J Neurol (1991) 238:409

\title{
Smoking and hypertension: risk factors for carotid stenosis
}

\author{
H. R. Mïller ${ }^{1}$ and M.W. Buser ${ }^{2}$ \\ ${ }^{1}$ Neurologische Universitätsklinik, Kantonsspital, Petersgraben 4, CH-4031 Basel, Switzerland \\ ${ }^{2}$ Universitätsrechenzentrum, Basel, Switzerland
}

J Neurol (1991) 238:97-102

An unfortunate error appeared in line 20 of the Summary.

This should read "... in the hypertensive smokers ..."

and not "in the hypertensive non-smokers". 